\title{
Functional characterization of novel mutations in the human cytochrome b gene
}

\author{
Frédéric Legros ${ }^{1}$, Evi Chatzoglou ${ }^{1}$, Paule Frachon ${ }^{1}$, Hélène Ogier de Baulny ${ }^{3}$, Pascal Laforêt ${ }^{1}$, \\ Claude Jardel $^{2}$, Catherine Godinot ${ }^{4}$ and Anne Lombès ${ }^{*, 1}$
}

${ }^{1}$ INSERM U523, Institut de Myologie, Hôpital de la Salpêtrière, 75651 Paris cedex 13, France; ${ }^{2}$ Biochimie B, Hôpital de La Salpêtrière, 75651 Paris cedex 13, France; ${ }^{3}$ Service de Neurologie, Hôpital Robert Debré, 75019 Paris; ${ }^{4}$ UMR5534 CGMC Université Cl Bernard, Lyon I, France

The great variability of the human mitochondrial DNA (mtDNA) sequence induces many difficulties in the search for its deleterious mutations. We illustrate these pitfalls by the analysis of the cytochrome b gene of 21 patients affected with a mitochondrial disease. Eighteen different sequence variations were found, five of which were new mutations. Extensive analysis of the cytochrome b gene of 146 controls found 20 supplementary mutations, thus further demonstrating the high variability of the cytochrome b sequence. We fully evaluated the functional relevance of 36 of these 38 mutations using indirect criteria such as the nature of the mutation, its frequency in controls, or the phylogenetic conservation of the mutated amino acid. When appropriate, the mtDNA haplotype, the heteroplasmic state of the mutation, its tissue distribution or its familial transmission were also assessed. The molecular consequences of the mutations, which appeared possibly deleterious in that first step of evaluation, were evaluated on the complex III enzymological properties and protein composition using specific antibodies that we have generated against four of its subunits. Two original deleterious mutations were found in the group of seven patients with overt complex III defect. Both mutations (G15150A (W135X) and T15197C (S151P)) were heteroplasmic and restricted to muscle. They had significant consequences on the complex III structure. In contrast, only two homoplasmic missense mutations with dubious clinical relevance were found in the patients without overt complex III defect. European Journal of Human Genetics (2001) 9, 510-518.

Keywords: Mitochondrial DNA; cytochrome b; complex III; human; mitochondrial diseases

\section{Introduction}

The human mitochondrial oxidative phosphorylation pathway consists of 13 subunits encoded by mitochondrial DNA (mtDNA) and at least 73 encoded by nuclear DNA. In addition, a very large number of nuclear genes are also indirectly involved in the respiratory chain function: chaperons, assembly factors, or enzymes involved in cofactor metabolism. ${ }^{1}$ The first step in genetic investigations of human mitochondrial disorders is to determine which genome, nuclear or mitochondrial, is responsible for the

\footnotetext{
*Correspondence: A Lombès, INSERM U523, Institut de Myologie, Hôpital de La Salpêtrière, 75651 Paris cedex 13; France;

E-mail: a.lombes@myologie.chups.jussieu.fr.

Received 9 February 2001; revised 26 April 2001; accepted 2 May 2001
}

disease. Direct analysis of the very numerous candidate nuclear genes is not possible. On the other hand, the high level of spontaneous mtDNA mutations ${ }^{2}$ is responsible for a wide diversity in the sequence of the mtDNA genes ${ }^{3,4}$ that renders difficult the functional evaluation of sequence variations in patients.

Ubiquinol: cytochrome c oxidoreductase (complex III or cytochrome $\mathrm{bc}_{1}$ complex, EC 1.10.2.2) is the simplest example of a respiratory complex with a double genetic origin as it is made up of 11 subunits, only one of which, the cytochrome $b$, is encoded by the mtDNA. Cytochrome $\mathrm{b}$ forms, with cytochrome $c_{1}$ and the Rieske iron-sulphur protein, the catalytic core of the cytochrome $b_{1}$ complex. Significant changes in its amino acid sequence have therefore a high probability to induce significant alteration of the catalytic function of complex III. Defects of the 
cytochrome $\mathrm{bc}_{1}$ complex are however rarely encountered in human mitochondrial disorders. They represented $2 \%$ of the respiratory chain alterations in the patients from

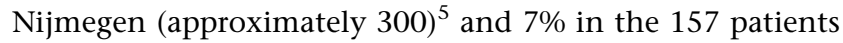
from Paris (Necker hospital). ${ }^{6}$ We have found a similar frequency (4\%) as, over the past 7 years, we have diagnosed seven cases with isolated complex III in more than 160 patients with respiratory chain defects (Dr A Lombès, unpublished results).

Contrasting with the wealth of information provided by the analysis of cytochrome $b$ mutants in yeast and bacteria, ${ }^{7-10}$ rare alterations of the human cytochrome $b$ gene have been associated with an overt isolated complex III defect $^{5,11-17}$ and their molecular consequences have only been analysed in three patients. ${ }^{11,15,17,18}$ All these mutations were heteroplasmic ie they coexisted with a residual amount of wild-type mtDNA. Half of them led to a truncated cytochrome $b$ protein. The deleterious potential of the other mutations has been ascertained on their absence in controls, on their skewed tissue distribution in patients and/or on the phylogenetic conservation of the modified amino acid. On the other hand, homoplasmic mutations of the human cytochrome $\mathrm{b}$ gene, ie mutations present in $100 \%$ of the mtDNA molecules, have been considered deleterious but they have not been associated with a defective complex III and their pathogenicity was only deduced from indirect criteria such as the phylogenetic conservation of the modified amino acid and control population screening. ${ }^{19-21}$ In addition, numerous silent cytochrome b sequence variants have been described and added as polymorphisms to the MITOMAP database of human mtDNA. ${ }^{4}$ Their identification as polymorphisms may however be considered disputable as they were often described in patients referred for a suspicion of a mitochondrial disease and who may have had variable defect of their complex III activity. ${ }^{22}$

In order to evaluate the role of the cytochrome $b$ gene in patients with a mitochondrial disease, we undertook the analysis of the human cytochrome b gene in 21 patients, seven with an overt isolated complex III defect and 14 without this enzymatic defect but with a disease pointing to an mtDNA dysfunction. ${ }^{23}$ We also determined the sequence variability of the cytochrome $b$ gene in a group of 146 controls. Numerous mutations, several of which were new mutations, were found both in the patients and the control group, thus demonstrating the high variability of the human cytochrome b sequence. Evaluation of the functional relevance of the sequence variants was first based on indirect criteria such as the nature of the mutation, its frequency in controls, or the phylogenetic conservation of the mutated amino acid. When appropriate, the mtDNA haplotype, the heteroplasmic state of the mutation, its tissue distribution or its familial transmission and the molecular consequences on the cytochrome $\mathrm{bc}_{1}$ complex enzymological characteristics and protein composition were analysed.

\section{Patients and methods \\ Patients}

All investigations were performed after the patient or the parents of the patient had given written informed consent according to the rules in our Institution. The cytochrome $b$ gene was analysed in an affected tissue from the patients and in leukocytes from the controls. The ethnical background of the patients and the controls is very diverse reflecting the cosmopolitan nature of the Paris urban area.

A first group of seven patients had an isolated cytochrome $\mathrm{bc}_{1}$ complex defect. The patients' age at the onset of the disease varied from birth to adolescence. The spectrum of affected organs was diverse: liver only (one patient), muscle only (two patients) or multisystemic (four patients with brain involvement). Four patients had consanguineous parents and two of them had similarly affected siblings. All patients had a significant and isolated complex III defect in muscle (five patients) or liver (two patients). The defect could be demonstrated in both muscle and skin fibroblasts in two patients with a generalised disease.

A second group of 14 patients had a mitochondrial disorder without an isolated complex III defect. They were included in the study because a genetic alteration of mtDNA was considered to be a likely cause of their disease for several of the following reasons: they had a typical mitochondrial accumulation in muscle with ragged-red fibres (seven patients); analyses of their respiratory chain disclosed mild and combined alterations of the respiratory complexes (eight patients); similarly affected relatives were noted in the family of seven patients but the familial pedigrees were not sufficient to assess the mode of inheritance. The patients' phenotypes were diverse in the age at onset of the disease (from birth to 40 years of age) and in the spectrum of affected organs: muscle was affected in 11/14 patients and the disease was generalised in $9 / 14$ patients.

The control group comprised 146 healthy adult subjects who volunteered to participate in the genetic analysis of autosomal dominant diseases. All these subjects were deemed to be free of any abnormal symptoms.

\section{Mitochondrial DNA analyses}

Total DNA was extracted from muscle, liver, blood or cultured skin fibroblasts and Southern blot analysis was performed on muscle and liver total DNA as described by Moraes et al. ${ }^{24}$

Cytochrome b gene analysis The patients' cytochrome b gene was sequenced in two overlapping fragments amplified by PCR (Table 1). Direct sequencing of the PCR fragments was performed using dye-terminator cycling sequencing on an automated sequencer according to the manufacturer (Abi Prism 377 from Perkin-Elmer Applied Biosystems).

Denaturing Gradient Gel Electrophoresis (DGGE) ${ }^{25}$ analysed the cytochrome $b$ gene in the control group. It was performed in five overlapping fragments (Table 1) according to Fischer and Lerman $^{26}$ that were mixed with DNA 
Table 1 Experimental conditions for the analysis of cytochrome b gene

\begin{tabular}{|c|c|c|c|}
\hline & 5' Primer & 3' Primer & Annealing $T^{r e}$ \\
\hline $5^{\prime}$ portion of cyt b gene & $14667-14687$ & $15289-15269$ & $58^{\circ} \mathrm{C}$ \\
\hline $3^{\prime}$ portion of cyt $b$ gene & $15189-15209$ & $15941-15921$ & $58^{\circ} \mathrm{C}$ \\
\hline \multicolumn{4}{|l|}{ Mispairing $P C R$} \\
\hline G15150A mutation & $15052-15072$ & $\begin{array}{c}15171-15151 \\
\text { (A15151G) }\end{array}$ & $54^{\circ} \mathrm{C}$ \\
\hline \multicolumn{4}{|l|}{$D G G E$} \\
\hline Fragment 1 (14700-14820) & $14636-14656(G C)$ & $14820-14800$ & $54^{\circ} \mathrm{C}$ \\
\hline Fragment $2(14800-15060)$ & $14788-14808$ & $15113-15093(P)$ & $58^{\circ} \mathrm{C}$ \\
\hline Fragment $3(15050-15380)$ & $14905-14930$ (GC) & $15480-15460$ & $52^{\circ} \mathrm{C}$ \\
\hline Fragment $4(15380-15690)$ & $15288-15307$ (GC) & $15694-15674$ & $56^{\circ} \mathrm{C}$ \\
\hline Fragment $5(15640-15940)$ & $15288-15307$ (GC) & $15941-15921$ & $57^{\circ} \mathrm{C}$ \\
\hline
\end{tabular}

All nucleotides are numbered according to Anderson ${ }^{27}$. Amplifications were performed with a one-minute denaturing step at $94{ }^{\circ} \mathrm{C}$, a oneminute annealing step, and finally a one-minute extension step at $72^{\circ} \mathrm{C}$. Primers: mismatched nucleotides are indicated between brackets. (GC), (P): addition of a GC clamp or of a psoralen molecule respectively at the $5^{\prime}$ end of the primer. DGGE: the portion of the amplified fragments that is analysed during the denaturing gradient gel electrophoresis (DGGE) is indicated between brackets.

fragments with known Anderson's sequence ${ }^{27}$ and denatured by heat prior to the DGGE.

Mispairing PCR was used to introduce a new restriction site for BsiWI or $K p n I$ in the presence of mutation G15150A or T15197C respectively (Table 1 ). To evaluate the proportion of the mutation, the DNA fragments containing the mutation were radiolabelled during the last cycle of amplification, ${ }^{28}$ digested with the appropriate enzyme, and separated by electrophoresis in 10\% acrylamide gels (acrylamide/bisacrylamide=19/1). The dried gels were counted in a $\beta$ imager.

Haplotype definition of mtDNA Restriction fragment length polymorphisms were used to define caucasian haplotypes of the mtDNA by the analysis of nine polymorphic sites as described by Torroni et al. ${ }^{29}$

\section{Biochemical analyses of complex III}

Enzymological analysis of complex III Complex III activity was analysed by spectrophotometry as the antimycin sensitive ubiquinol cytochrome c oxidoreductase. ${ }^{30}$ A value below the normal range with normal activity of all the other respiratory complexes indicated an isolated complex III defect.

Antimycin and myxothiazol are representative of each class of known inhibitors of complex III, with different sites of fixation located on each side of the internal mitochondrial membrane. ${ }^{7}$ Kinetic parameters were determined at various concentrations of the substrate (decylubiquinol from Sigma), and of myxothiazol (Sigma) and antimycin (Sigma).

Western blot analysis of complex III subunits Antibodies against core 1 , core 2 , the Rieske iron-sulphur protein, and cytochrome $c_{1}$ were obtained in rabbits by immunisation with the subunits separated by SDS-PAGE from isolated beef heart complex III. ${ }^{31}$ Antibodies against cytochrome b were obtained by immunization with a synthetic peptide (corresponding to amino acids 260 to 272 of cytochrome b) bound to Keyhole Limpet hemocyanine followed by immunopurification of the serum. Antibodies against subunits COX2 and COX4 of the cytochrome c oxidase (complex IV of the respiratory chain) were used as controls for the amount of mitochondrial proteins. ${ }^{32}$

Mitochondrial proteins from muscle were obtained by homogenisation of muscle followed by differential centrifugations to obtain the crude mitochondrial pellet. Electrophoresis and immunoblotting procedures were performed as described by Lombès et al. ${ }^{33}$ Primary antibody concentrations were $1 / 50000$ for anti-core 1 , anti-core 2 , and anti-ISP protein, 1/1000 for anti-cytochrome $c_{1}, 1 / 400$ for anticytochrome b, 1/1000 for anti-COX2 and 1/500 for antiCOX 4 in PBS $+0.1 \%$ Tween $20,1 \%$ BSA and $2 \%$ skimmed milk. Secondary antibodies were peroxidase-conjugated antirabbit antibodies from Dako. Specific binding was revealed by chemiluminescence using the $\mathrm{ECL}^{\mathrm{m}}$ system (Amersham) according to the manufacturer's instructions.

\section{Results}

Thirty-eight sequence variations in the cytochrome b gene were identified in the patients and in the control population

The size and the amount of the mtDNA from muscle and liver were found to be normal by Southern blot analysis in all patients (not shown). Direct sequencing of the cytochrome $b$ gene of the patients disclosed 18 sequence variations that are listed in Table 2. 
Table 2 Sequence variations of the patients' cytochrome b gene

\begin{tabular}{|c|c|c|c|c|c|c|}
\hline \multicolumn{2}{|c|}{ Mutation } & \multirow[b]{2}{*}{ \# (/21 patients) } & \multirow[b]{2}{*}{ \# (/146 controls) } & \multirow[b]{2}{*}{ MtDNA haplotype } & \multicolumn{2}{|c|}{ Phylogenetic study } \\
\hline Nucleotide & Amino acid & & & & Identity: \% & AA usage: $\%$ \\
\hline T14766C & I7L & 9 & $74(51 \%)$ & ${ }^{*}, \mathrm{H}$ & 2 & $\mathrm{~S}+\mathrm{T}: 85$ \\
\hline T14793C & $\mathrm{H} 16 \mathrm{R}$ & 3 & $6(4 \%)$ & * & 22 & D+E: 29, N: 27, H: 14 \\
\hline T14798C & F18L & 2 & $15(10 \%)$ & $\mathrm{J}, \mathrm{K}$ & 44 & $\mathrm{~L}+\mathrm{I}+\mathrm{V}: 54$ \\
\hline G15043A & G99G & 1 & $13(9 \%)$ & - & & \\
\hline G15148A & P134P & 1 & 0 & - & & \\
\hline G15150A (H) & W135X & 1 & 0 & J & 99 & \\
\hline T15197C (H) & S151P & 1 & 0 & $\mathrm{H}$ & 99 & \\
\hline G15217A & G157G & 1 & 0 & - & & \\
\hline A15218G & T158A & 1 & $4(3 \%)$ & * & 34 & Q+N: 45, D+E: 13 \\
\hline G15257A & D171N & 1 & 0 & $\mathrm{~K}$ & 90 & \\
\hline G15301A & L185L & 2 & $7(5 \%)$ & - & & \\
\hline
\end{tabular}

Nucleotides are numbered according to Anderson. ${ }^{27}$ Original mutations are in bold characters. (H): Mutation appearing heteroplasmic following DGGE. Amino acids are designated by the single letter amino acid code, syn=synonymous mutation, X: stop codon. MtDNA haplotype $\mathrm{K}, \mathrm{J}$, and $\mathrm{H}$ are defined respectively by the loss of 9052/Haell, the loss of both 13704/Bst NI and 16065/Hinfl and the loss of both 10394/Ddel and 7025/Alul restriction sites. ${ }^{29}$ Haplotype $\mathrm{H}$ was found in six patients, haplotypes $\mathrm{K}$ and J were found in one patient each. The African outgroup (Af), defined by the conservation of all these restriction sites, was found in three patients. The haplotype * defined by the loss of 10394/Ddel and the conservation of 7025/Alul restriction sites, was found in seven patients. The phylogenetic study is based on the sequences reported from 112 species. $^{7}$ Identity gives the percentage of species with an aminoacid identical to the human sequence. Amino acid usage gives the percentage of the aminoacid or groups of amino acids most often encountered at that position in the analysed species.

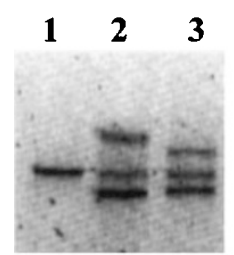

14700-14800

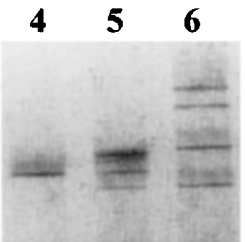

14800-15060

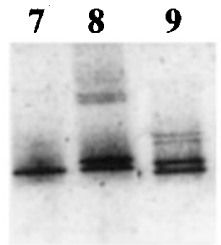

15050-15380

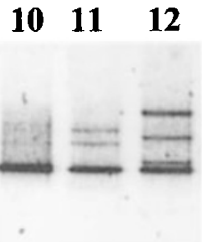

15380-15690
131415

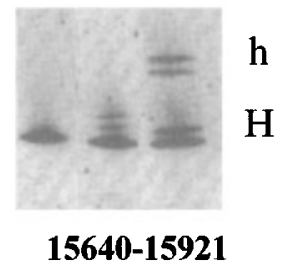

Figure 1 Analysis with denaturing gradient gel electrophoresis (DGGE) of amplified fragments of the cytochrome b gene. The mitochondrial DNA sequence fragment analysed by the DGGE procedure is indicated below each example. Migration pattern of DNA fragment with Anderson's consensus sequence is shown in lanes 1, 4, 7, 10 and 13. Migration pattern of DNA fragments differing by one base from the Anderson consensus sequence, mixed with DNA fragments with Anderson's sequence, and denatured prior to their analysis by DGGE is shown on lanes 2, 3, 5, 6, 8, 9, 11, 12, 14, 15 (mutations T14783C, T14766C, T14798C, G15043A, G15301A, A15326G, T15394C, C15452A, T15748C, A15607G, respectively). The level of migration of the homoduplex molecules (H) and heteroduplex molecules $(h)$ is indicated on the right hand side of the figure. While the difference in migration level may not be obvious between two species of homoduplex molecules (see lane 14), the retardation of the heteroduplex molecules is always readily visible in the upper part of the gel.

All these sequence variations were easily detected by denaturing gradient gel electrophoresis (DGGE) when the DNA variants were mixed with DNA fragments with known Anderson's sequence ${ }^{27}$ and denatured by heat prior to the DGGE (Figure 1). We therefore used DGGE to perform the extensive screening of the cytochrome $b$ gene of 146 controls. The absence of heteroduplex molecules in the DGGE demonstrated that the two DNA fragments present in the mix had an identical sequence. The presence of heteroduplex molecules was followed by direct sequencing of the cytochrome $b$ gene in its half encompassing the fragment analysed by DGGE. Using that protocol, 140 sequences were determined. They identified numerous variants that are listed in Table 3 . Altogether, 38 variations in the cytochrome $b$ sequence were identified both in the patients and in the control population (Tables 2 and 3). 
Table 3(a) Sequence variations of the cytochrome b gene found only in controls

\begin{tabular}{|c|c|c|c|c|}
\hline \multicolumn{2}{|c|}{ Mutation } & \multirow{2}{*}{$\begin{array}{c}\#(/ 146 \\
\text { controls })\end{array}$} & \multicolumn{2}{|c|}{ Phylogenetic study } \\
\hline Nucleotide & Amino acid & & Identity: \% & AA usage: $\%$ \\
\hline G14905A & M53M & $4(3 \%)$ & & \\
\hline C15028T & L94L & $2(1 \%)$ & & \\
\hline T15097C & L117L & $2(1 \%)$ & & \\
\hline G15106A & L120L & $1(1 \%)$ & & \\
\hline C15201G & A152G & $1(1 \%)$ & 93 & \\
\hline A15226C & L160L & $2(1 \%)$ & & \\
\hline A15236G & I164V & $2(1 \%)$ & 59 & $\mathrm{~L}+\mathrm{I}+\mathrm{V}: 71, \mathrm{~A}: 28$ \\
\hline A15244G & G166G & $3(2 \%)$ & & \\
\hline T15262A & S172R & $2(1 \%)$ & 3 & $\mathrm{~N}: 71, \mathrm{~K}: 25$ \\
\hline G15314A & A190T & $1(1 \%)$ & 60 & $\mathrm{~T}+\mathrm{S}: 20, \mathrm{~L}+\mathrm{I}+\mathrm{V}: 16$ \\
\hline T15394C & D216D & $1(1 \%)$ & & \\
\hline C15442A & L232I & $2(1 \%)$ & 9 & $\begin{array}{c}\mathrm{F}: 67, \mathrm{~L}+\mathrm{l}+\mathrm{V}: 19, \\
\mathrm{~A}+\mathrm{G}: 14\end{array}$ \\
\hline T15454C & L236L & $1(1 \%)$ & & \\
\hline T15601C & P285P & $1(1 \%)$ & & \\
\hline A15607G & K287K & $6(4 \%)$ & & \\
\hline A15662G & P305P & $1(1 \%)$ & & \\
\hline T15693C & M316T & $1(1 \%)$ & 25 & $\mathrm{~T}: 57$ \\
\hline T15748C & I334I & $1(1 \%)$ & & \\
\hline A15775G & V343V & $1(1 \%)$ & & \\
\hline G15803A & V353I & $1(1 \%)$ & 28 & $L+l+V: 91$ \\
\hline
\end{tabular}

(b) Spectrophotometric analyses of complex III activities in the tissues from the patients with a heteroplasmic mutation of the cytochrome $b$ gene

\begin{tabular}{|c|c|c|c|c|c|c|}
\hline \multirow[b]{2}{*}{ Tissue } & \multicolumn{2}{|c|}{ Muscle } & \multicolumn{2}{|c|}{$\begin{array}{c}\text { Blood } \\
\text { lymphocytes }\end{array}$} & \multicolumn{2}{|c|}{ Skin fibroblasts } \\
\hline & $\%$ mut & III & $\%$ mut & III & $\%$ mut & III \\
\hline G15150A & 60 & 7 & 0 & 22 & 0 & 55 \\
\hline T15197C & 80 & 17 & 0 & 28 & 0 & 50 \\
\hline Controls & 0 & $\begin{array}{c}103 \pm 27 \\
(58-194)\end{array}$ & 0 & $\begin{array}{c}39 \pm 13 \\
(18-68)\end{array}$ & 0 & $\begin{array}{c}30 \pm 6 \\
(26-52)\end{array}$ \\
\hline $\mathrm{M} \pm \mathrm{SD}$ (range) & & $n=180$ & & $n=21$ & & $n=24$ \\
\hline
\end{tabular}

III=antimycin sensitive activity of the respiratory complex III expressed in nanomoles cytochrome c oxidized per minute and milligram of protein; \% mut=proportion of the mutation in the tissue; control values are shown as mean \pm standard deviation followed by the range of values between brackets and by the number of control samples $(n)$.

\section{Evaluation with indirect criteria allowed determining the functional relevance of 36 of the 38 sequence variations}

Half of the cytochrome $b$ gene sequence variations (19/38) had never been reported and most are restricted to few patients and/or to less than $2 \%$ of the controls (Tables 2 and 3). In order to distinguish between polymorphisms and potentially deleterious mutations, we used indirect criteria such as the heteroplasmic state of the mutation, its nature (silent or replacement), its frequency in our control population, the importance of the induced amino acid change and the phylogenetic conservation of the mutated amino acid. We also established the mtDNA haplotype for most sequence variations of patients. All these parameters are indicated in Tables 2 and 3.

From the DGGE pattern, 36 sequence variations looked homoplasmic. Twenty of them were synonymous mutations (Tables 2 and 3). Fourteen were missense mutations but four of them (T14766C, T14798C, A15326G and C15452A) were frequently encountered in our control population and the amino acid change induced by the remaining 10 was considered to be very mild because either the modified amino acid or an amino acid with similar physicochemical characteristics was commonly encountered at that position in other animal species (T14793C, G15110A, C15201G, A15218G, A15236G, T15262A, G15314A, C15442A, T15693C and G15803A). Furthermore, mutation T14793C was found in three patients and segregated with a specific Caucasian haplogroup (* in Table 2) defined by the loss of 10394/DdeI and the conservation of $7025 / A l u I$ restriction sites. Thirtyfour of the homoplasmic mutations could therefore be classified as polymorphisms but two homoplasmic missense mutations (A14769G and G15257A) remained disputable. They were found in only one patient thus preventing the analysis of their segregation with mtDNA haplogroups. They were absent in the control population. Mutation A14769G (N8T) has been reported as a polymorphism. ${ }^{4}$ It was found in liver and fibroblasts from a newborn patient who had hyperlactatemia and hepatomegaly with an isolated mild complex III defect in liver. The threonine introduced by the mutation has physicochemical characteristics quite different from histidine (present in $88 \%$ of the species) and arginine (present in $10 \%$ of the species). Mutation G15257A (D171N) changes a highly conserved aminoacid residue. It has been reported as being potentially deleterious in association with Leber Hereditary Optic Neuropathy but appeared confined to the J mtDNA haplogroup. ${ }^{21}$ It was found in a 5 years old child with motor and development delay without symptoms of Leber Hereditary Optic Neuropathy nor relatives with that disease. Muscle investigations showed predominance of type I fibres and high levels of citrate synthase without significant defect of any of the respiratory chain complexes. The patient has the $\mathrm{K}$ mtDNA haplotype.

From the DGGE pattern, two mutations (G15150A and T15197C) looked heteroplasmic. Both mutations were absent in the control population. Mutation G15150A was nonsense. Mutation T15197C changed serine 151 to a proline, which constitutes an important modification in the physicochemical properties of the amino acid. Furthermore, amino acid position 151 is a serine in 68 species, it is replaced by a threonine in only one species. The predicted structure of the mutated protein, according to Levitt, showed destabilisation of the $\alpha$-helix cd2 that is in close relationship with the catalytic centre or $_{\mathrm{p}}$ of the cytochrome b protein. ${ }^{7,34}$ 
Molecular consequences of the mutations

Homoplasmic mutations G15257A and A14769G The activity of complex III and its sensitivity for both antimycin and myxothiazol were similar to control values in cultured skin fibroblasts that had mutation A14769G or G15257A in a homoplasmic state. This finding cannot exclude some deleterious impact in other tissues or in particular metabolic circumstances.

Heteroplasmic mutations G15150A and T15197C Radioactive mispairing PCR was used to precisely evaluate the proportion of the mutations in diverse tissues of the patients and in the blood of the mother of the patient with the G15150A mutation (Figure 2). Both mutations were restricted to muscle tissue where they represented a majority of the mtDNA molecules (60 and 80\% for mutation G15150A and T15197C respectively) (lanes Muscle in Figure 2). They were absent in blood leukocytes, skin fibroblasts and/or lymphoblastoid cell line (lanes Blood, Fibroblasts and Lymph cells in Figure 2). Interestingly both patients had an isolated intolerance to exercise from their late childhood. Their
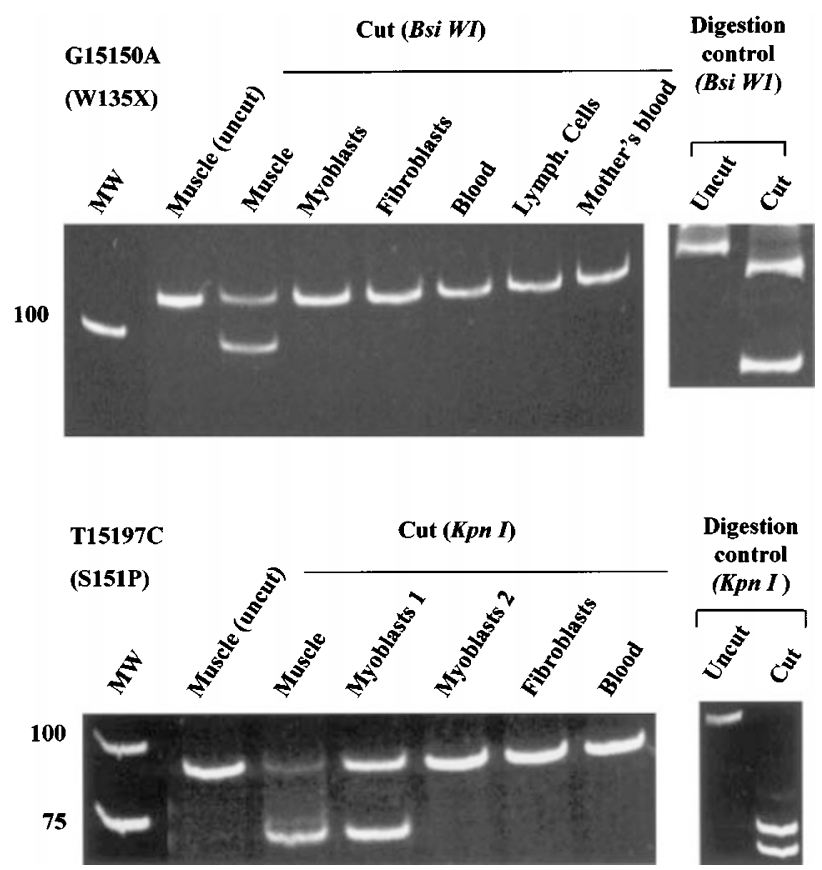

Figure 2 Analysis of the proportion of the mutations G15150A and T15197C by mispairing PCR and restriction. The presence of the mutation introduces a new restriction site for the enzyme. The tissue from which the DNA has been extracted is indicated on the top of the lanes; (uncut)=fragments that have not been digested by the restriction enzyme; cut=fragments digested by the restriction enzyme indicated between brackets. Digestion control: efficiency of the digestion was tested on another fragment of the mtDNA having a natural restriction site for the enzyme. $M W=$ size standards in base pairs, their size in base pairs is indicated on the left hand side of the gels. complex III was severely defective in muscle but normal in leukocytes and skin fibroblasts. Despite the presence of uridine and pyruvate supplementing the culture medium, mutation G15150A was undetectable in the myoblasts obtained from the patients' muscle (lane Myoblasts Figure 2) while mutation $\mathrm{T} 15197 \mathrm{C}$ was present in $50 \%$ of the mtDNA molecules extracted from the myoblasts pellet obtained after one passage (lane Myoblasts 1 Figure 2) but was undetectable in the pellet obtained after a second passage (lane Myoblasts 2 Figure 2). The familial transmission could be analysed only for mutation G15150A, which was absent from the blood of the patient's mother. However, both patients had a negative family history.

Spectrophotometric analysis of the respiratory chain showed that the complex III activity was extremely decreased in the muscle with a high proportion of one of the mutations whereas the other respiratory chain activities were normal or elevated (Table 3 ). In both muscles, the proportion of the total complex III activity that was antimycin sensible was normal (control values $=85 \pm 10 \%$ ). Kinetic analysis of the complex III was performed in the muscle with mutation T15197C but not in the case of mutation G15150A because of the very limited amount of tissue. Analysis of the influence of the decylubiquinol concentration showed an overall severe reduction in the reaction rate in a substrate concentration range from 0.66 to $21.15 \mu \mathrm{M}$ decylubiquinol (Figure 3A) but the apparent $\mathrm{Km}$ appeared to be similar to the values found in control muscles. Complete resistance to myxothiazol was present (Figure 3B) and contrasted with a normal sensitivity to antimycin (Figure 3C).

Western blot analysis of the cytochrome $\mathrm{bc}_{1}$ complex in muscle showed that the presence of the nonsense mutation G15150A was associated with an undetectable cytochrome b protein. Core 1 and iron sulphur protein (ISP) were greatly diminished, but Core 2 and cytochrome $c_{1}$ were relatively spared (Figure 4). In contrast, the presence of the missense mutation T15197C was associated with a reduced amount of cytochrome $\mathrm{b}$ and cytochrome $\mathrm{c}_{1}$ while the amount of Core 1, ISP and Core 2 appeared normal (Figure 4). The two complex IV subunits used as controls (COX2 as control for the mtDNA-encoded proteins and COX4 as control for the nuclear DNA-encoded proteins) were normal in both muscles. The two heteroplasmic mutations were therefore associated with significant enzymatic and protein abnormalities that underline their pathogenic relevance.

\section{Discussion}

Identification of the two severely deleterious mutations G15150A (W135X) and T15197C (S151P) in the cytochrome $\mathrm{b}$ gene further confirmed that cytochrome $\mathrm{b}$ alterations cause isolated complex III defect in humans.,11-17 These two deleterious cytochrome b mutations were heteroplasmic and had a very skewed tissue distribution resembling that observed in eight of the 12 reported cytochrome b 
A

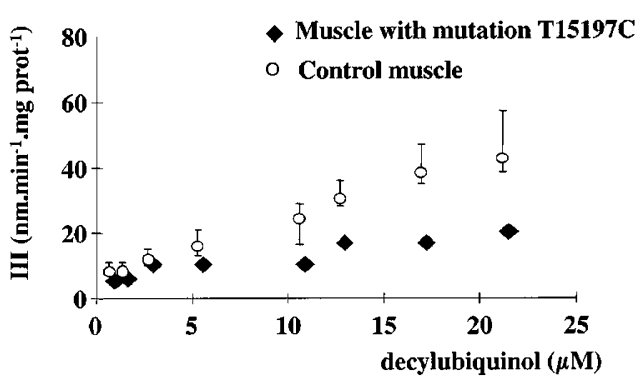

B

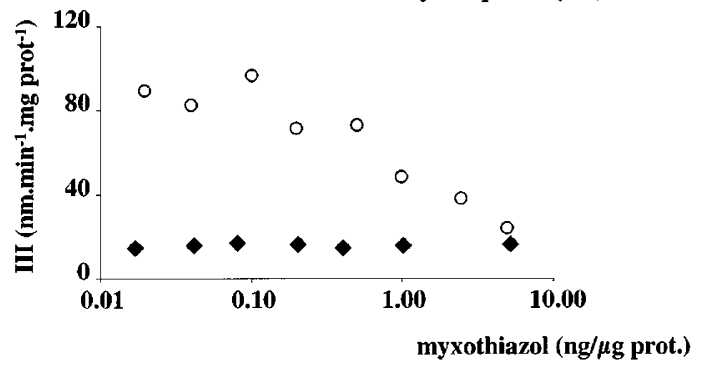

C

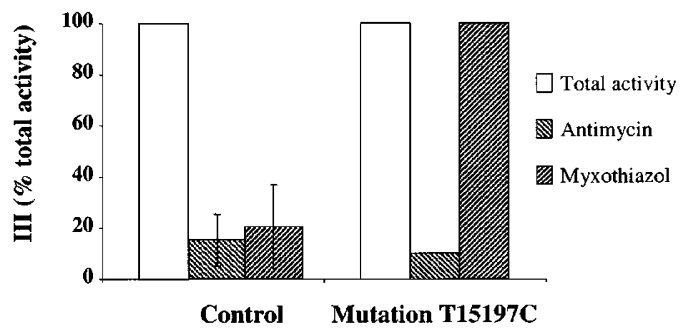

Figure 3 Kinetic analysis of complex III activity in the muscle with mutation T15197C in $80 \%$ of the mtDNA molecules. Rate of complex III reaction in the presence of increasing concentration of decylubiquinol (A) or in the presence of increasing concentration of myxothiazol (B). Reaction rate is expressed in nanomoles cytochrome $c$ reduced per minute and per milligram protein, decylubiquinol concentration is in micromoles/L $(\mu \mathrm{M})$ and myxothiazol concentration is in nanograms per micrograms of protein. C: Antimycin sensitive (Antimycin) and myxothiazol sensitive (Myxothiazol) activities expressed as \% of total activity (without inhibitors) in controls and in the muscle with mutation T15197C. Control values are $85 \pm 10(n=100)$ and $80 \pm 16 \%(n=8)$ for the antimycin sensitive and the myxothiazol sensitive activities respectively.

mutations. ${ }^{11,12,14,16,35}$ How these mutations may accumulate to such a high proportion in muscle and be, at the same time, segregated efficiently in all other organs remains to be established. In any case, this finding emphasises the limited significance of data from mtDNA extracted from unaffected tissue such as blood leukocytes.

Important insight on the complex III structure was brought by the analysis of the molecular consequences of the two deleterious mutations. Mutation G15150A (W135X) resulted in the absence of cytochrome $b$ protein, a global disruption of the complex III but with a relative preservation of core 2 and cytochrome $c_{1}$ as compared to core 1 and ISP. Darley Usmar et $a l^{18}$ found similar alterations in muscle mitochondria from a

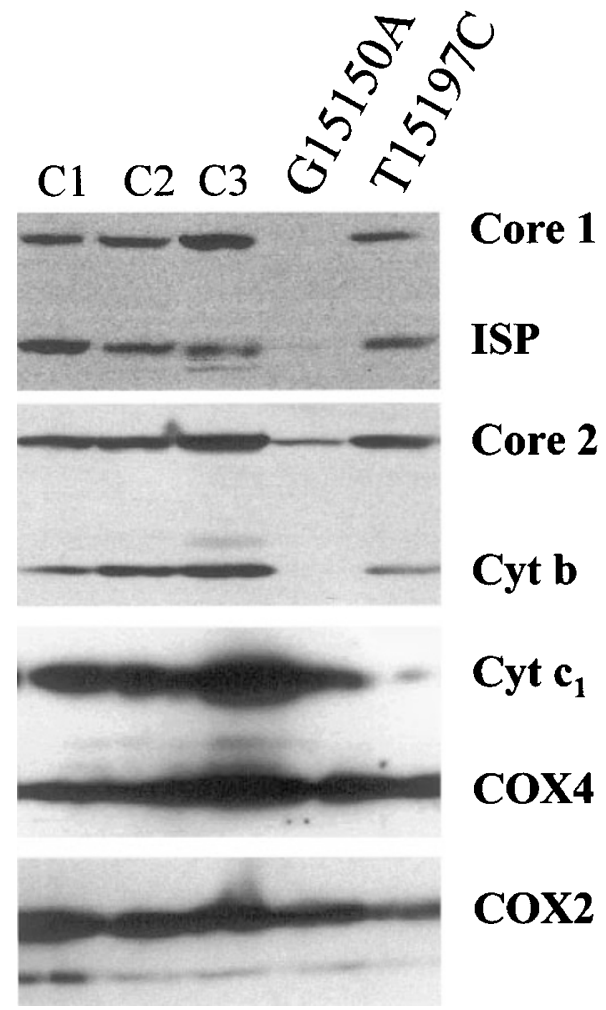

Figure 4 Western blot analysis of the cytochrome bc1 complex in muscle with the mutation G15150A (W135X) and T15197C (S151P). $C_{1}, C_{2}, C_{3}=$ mitochondrial proteins from three different control muscles. Core 1, Iron Sulphur Protein (ISP), Core 2, cytochrome b (Cyt b) and cytochrome $c_{1}$ (cyt $\left.c_{1}\right)=$ subunits of the cytochrome bc 1 complex. COX2 and COX4=subunits of the cytochrome c oxidase (complex IV of the respiratory chain).

patient with an isolated complex III defect and postulated, at that time, a null mutation of the cytochrome $b$ gene. Indeed, mutation G15242A (G165X) was recently identified in the muscle from this patient. ${ }^{17}$ Analysis of these two patients shows therefore the great similarity of the molecular consequences between a nonsense mutation in the cytochrome $b$ gene in humans and the cytochrome $b$ null mutants described in yeast including the relative preservation of the cytochrome $c_{1}$ as compared to ISP. ${ }^{36-38}$ The molecular consequences of mutation T15197C (S151P) cannot be compared directly with yeast mutant because, to our knowledge, serine ${ }_{151}$ has never been mutated in yeast. However, numerous mutants have been described in the neighbouring amino acids and have demonstrated the critical role of individual amino acids in that region in the resistance to myxothiazol and the catalytic activity of center $\mathrm{Q}_{\mathrm{p}}$ in yeast and in bacteria. $^{7-9,39,40}$ The slightly reduced amount of the cytochrome $b$ and cytochrome $c_{1}$ proteins with normal amount of the core proteins and ISP is more difficult to interpret in the absence of similar analysis in yeast. The significant destabilisation of the $\alpha$-helix cd 
predicted in the mutated protein may be an important factor in the major effect of the mutation T15197C.

The general relevance for human cytochrome $b$ of the data brought by the yeast mutants has also been shown by mutation G15615A (G290D), ${ }^{11}$ which was associated with antimycin resistance and instability of the cytochrome $\mathrm{bc}_{1}$ complex similar to that observed with the neighbouring mutations G340E and L282F (in the yeast sequence). ${ }^{7,41}$ Only one recently reported mutation: G15243A (G166E) ${ }^{15}$ does not fit with data from yeast mutants. The mutation has been associated with a very slow rate of reaction and instability of the cytochrome $b_{1}$ complex in the presence of lauryl maltoside but mutation of a neighbouring amino acid in yeast (G167R) had no functional consequence on the rate of the reaction. ${ }^{42}$

The two homoplasmic missense mutations with dubious clinical relevance, A14769G (N8T) and G15257A (D171N), exemplify the pitfalls of the functional assessment of rare and potentially mildly deleterious mtDNA mutations. The amino acid replacement and the phylogenetic conservation of the amino acid position were significant. The mutations were absent in the control population. The mtDNA haplogroup were not useful as it had not been reported with mutation $\mathrm{A} 14769 \mathrm{G}^{22}$ and is closely related to that identified in our patient with mutation $\mathrm{G} 15257 \mathrm{~A}^{21,43}$ thus leaving open the possibility for the mutation to be a rare polymorphism. Subtle modifications in the kinetic behaviour of the complex III are likely to have been ignored considering the technical limitations of the complex III enzymologic analysis in cultured skin fibroblasts. No identical yeast mutant has been previously described. The relevance of mutations A14769G (N8T) and G15257A (D171N) will therefore have to wait until a sufficiently large group of controls and patients have been collected on the available databases.

In conclusion, extensive screening analysis of the cytochrome $b$ gene both in patients and in controls allowed the identification of two original deleterious mutations. Both mutations were heteroplasmic and associated with an overt and isolated complex III defect. In parallel, numerous homoplasmic variants were found in the cytochrome $b$ gene. Two of the 17 identified missense mutations had a potentially deleterious potential that was not definitely evaluated in that work and will have to wait for further population study.

Screening for deleterious cytochrome b mutations should therefore be done in patients with an isolated complex III defect, using DNA extracted from the defective tissue and a technical approach allowing the direct diagnosis of the heteroplasmic nature of the mutation (DGGE in our study). Follow-up investigations should include the analysis of the complex III enzymologic properties and protein composition, both being possible on minimal amount of tissue and allowing precise demonstration of the mutation's molecular consequences thus providing essential information as to the structure/function relationship of human complex III.

\section{Acknowledgments}

This work has been supported by a grant from La Fondation pour la Recherche Médicale and from the AFM (Association Française contre les Myopathies). Evi Chatzoglou was the recipient of a post-doctoral fellowship from AFM. We are very grateful to Dr Marc Fiszman, Dr Manuel Rojo and Dr Gillian Butler-Brown for careful reading of the manuscript and useful comments.

\section{References}

1 Attardi G, Schatz G: Biogenesis of mitochondria. Ann Rev Cell Biol 1988; 4: 289-333.

2 Brown WM, George M, Wilson AC: Rapid evolution of animal mtDNA. Proc Natl Acad Sci USA 1979; 76: 1967-1971.

3 Wallace DC: Mitochondrial DNA sequence variation in human evolution and disease. Proc Natl Acad Sci USA 1994; 91: 8739_ 8746.

4 Kogelnik AM, Lott MT, Brown MD, Navathe SB, Wallace DC: MITOMAP: an update on the status of the human mitochondrial genome database. Nucl Acids Res 1997; 25: 196-199.

5 Mourmans J, Wendel U, Bentlage HACM et al: Clinical heterogeneity in respiratory chain complex III deficiency in childhood. J Neurol Sci 1997; 149: 111-117.

6 von Kleist-Retzow JC, Cormier-Daire V, de Lonlay P et al: A high rate $(20 \%-30 \%)$ of parental consanguinity in cytochromeoxidase deficiency. Am J Hum Genet 1998; 63: 428-435.

7 Degli Esposti M, De Vries S, Crimi M, Ghelli A, Patarnello T, Meyer A: Mitochondrial cytochrome b: evolution and structure of the protein. Bioch Bioph Acta 1993; 1143: 243-271.

8 Gennis RB, Barquera B, Hacker B et al: The bc1 complexes of Rhodobacter speroides and Rhodobacter capsulatus. I Bioenerg Biomemb 1993; 25: 195-209.

9 Colson AM: Random mutant generation and its utility in uncovering structural and functional features of cytochrome b in Saccharomyces cerevisae. J Bioenerg Biomemb 1993; 25: 211 220.

10 Berry EA, Guergova-Kuras M, Huang L, Crofts AR: Structure and function of cytochrome bc complexes. Annu Rev Biochem 2000; 69: $1005-1075$.

11 Bouzidi MF, Carrier H, Godinot C: Antimycin resistance and ubiquinol cytochrome $\mathrm{c}$ reductase instability associated with a human cytochrome b mutation. Bioch. Biophem Acta 1996; 1217: $199-209$.

12 Andreu AL, Bruno C, Shanske S et al: Missense mutation in the mtDNA cytochrome $b$ gene in a patient with myopathy. Neurology 1998; 51: 1444-1447.

13 de Coo IFM, Renier WO, Ruitenbeek W et al: A 4-base pair deletion in the mitochondrial cytochrome $\mathrm{b}$ gene associated with Parkinsonism/MELAS overlap syndrome. Ann Neurol 1999; 45: $130-133$.

14 Andreu AL, Hanna MG, Reichmann $\mathrm{H}$ et al: Exercise intolerance due to mutations in the cytochrome $\mathrm{b}$ gene of mitochondrial DNA. N Engl J Med 1999; 341: 1037 - 1044.

15 Valnot I, Kassis J, Chretien D et al: A mitochondrial cytochrome $\mathrm{b}$ mutation but no mutations of nuclearly encoded subunits in ubiquinol cytochrome c reductase (complex III) deficiency. Hum Genet 1999; 104: 460-466.

16 Andreu AL, Checcarelli N, Iwata S, Shanske S, DiMauro S: A missense mutation in the mitochondrial cytochrome $b$ gene in a revisited case with histiocytoid cardiomyopathy. Pediatr Res 2000; 48: 311-314.

17 Keightley JA, Anitori R, Burton MD, Quan F, Buist NRM, Kennaway NG: Mitochondrial encephalomyopathy and complex III deficiency associated with a stop-codon mutation in the cytochrome b gene. Am J Hum Genet 2000; 67: 1400 - 1410 . 
18 Darley-Usmar VM, Kennaway NG, Buist NRM, Capaldi RA: Deficiency in ubiquinone cytochrome c reductase in a patient with mitochondrial myopathy and lactic acidosis. Proc Natl Acad Sci USA 1983; 80: 5103-5106.

19 Johns DR, Neufeld MJ: Cytochrome b mutations in Leber hereditary optic neuropathy. Biochem Biophys Res Comm 1991; 181: $1358-1364$

20 Oostra RJ, Bolhuis PA, Zorne-Ende I, de Kok-Nazaruk MM, Bleeker-Wagemakers EM: Leber's hereditary optic neuropathy: no significant evidence for primary or secondary pathogenicity of the 15257 mutation. Hum Genet 1994; 94: 265-270.

21 Brown MD, Torroni A, Reckord CL, Wallace DC: Phylogenetic analysis of Leber's hereditary optic neuropathy mitochondrial DNA's indicates multiple independent occurrences of the common mutations. Hum Mut 1995; 6: 311-325.

22 Andreu AL, Bruno C, Hadjigeorgiou GM, Shanske S, DiMauro S: Polymorphic variants in the human mitochondrial cytochrome b gene. Mol Genet Metab 1999; 67: 49-52.

23 Simon DK, Johns DR: Mitochondrial disorders: clinical and genetic features. Annu Rev Med 1999; 50: 111-127.

24 Moraes CT, Shanske S, Tritschler HJ et al: mtDNA depletion with variable tissue expression: a novel genetic abnormality in mitochondrial diseases. Am J Hum Genet 1991; 48: 492-501.

25 Myers RM, Maniatis T, Lerman LS: Detection and localization of single base changes by denaturing gel electrophoresis. Meth Enzymol 1987; 155: $501-527$.

26 Fischer SG, Lerman LS: DNA fragments differing by single basepair substitutions are separated in denaturing gradient gels: correspondence with melting theory. Proc Natl Acad Sci USA 1983; 80: $1579-1583$.

27 Anderson S, Bankier AT, Barrel BG et al: Sequence and organization of the human mitochondrial genome. Nature 1981; 290: 457-465.

28 Moraes CT, Ciacci F, Bonilla E et al: Two novel pathogenic mitochondrial DNA mutations affecting organelle number and protein synthesis. J Clin Invest 1993; 92: 2906-2915.

29 Torroni A, Lott MT, Cabell MF, Chen YS, Lavergne L, Wallace DC: MtDNA and the origin of Caucasians: identification of ancient Caucasian-specific haplogroups, one of which is prone to a recurrent somatic duplication in the D-Loop region. Am J Hum Genet 1994; 55: 560-576.

30 Birch-Machin MA, Shepherd IM, Watmough NJ et al: Fatal lactic acidosis in infancy with a defect of complex III of the respiratory chain. Pediatr Res 1989; 25: 553 - 559.

31 Schägger H, Link TA, Engel WD, von Jagow G: Isolation of the eleven protein subunits of the bc1 complex. Methods Enzymol 1986; 126: 224-237.
32 Possekel S, Lombès A, Ogier de Baulny $\mathrm{H}$ et al: Immunohistochemical analysis of muscle cytochrome c oxidase deficiency in children. Histochem 1995; 103: 59-68.

33 Lombès A, Mendell JR, Nakase $\mathrm{H}$ et al: Myoclonic epilepsy and ragged-red fibers with cytochrome c oxidase deficiency: neuropathology, biochemistry, and molecular genetics. Ann Neurol 1989; 26: 20-33.

34 Iwata S, Lee JW, Okada $\mathrm{K}$ et al: Complete structure of the 11subunit bovine mitochondrial cytochrome bc1 complex. Science 1998; 281: $64-71$.

35 Andreu AL, Bruno C, Dunne TC et al: A nonsense mutation (G15059A) in the cytochrome $b$ gene in a patient with exercise intolerance and myoglobinuria. Ann Neurol 1999; 45: 127 - 130.

36 Capeillere-Blandin C, Ohnishi T: Investigation of the iron-sulfur clusters in some mitochondrial mutants of saccharomyces cerevisae. Eur J Biochem 1982; 122: 403 - 413

37 Sen K, Beattie DS: Decreased amounts of core proteins I and II and the iron-sulfur protein in mitochondria from yeast lacking cytochrome $\mathrm{b}$ but containing cytochrome $\mathrm{c}_{1}$. Arch Biochem Biophys 1985; 242: 393-401.

38 di Rago JP, Macadre C, Lazowska J, Slonimski PP: The C-terminal domain of yeast cytochrome $b$ is essential for a correct assembly

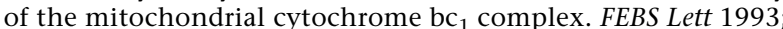
328: $153-158$

39 Geier BM, Schägger H, Brandt U, Colson AM, Von Jagow G: Point mutation in cytochrome $b$ of yeast ubihydroquinone:cytochrome-c oxidoreductase causing myxothiazol resistance and facilitated dissociation of the iron-sulfur subunit. Eur J Biochem 1992; 208: $375-380$.

40 Bruel C, di Rago JP, Slonimski PP, Lemesle-Meunier D: Role of the evolutionarily conserved cytochrome $b$ tryptophan 142 in the ubiquinol oxidation catalysed by the $\mathrm{bc}_{1}$ complex in the yeast Saccharomyces cerevisae. J Biol Chem 1995; 270: 22321 22328.

41 Lemesle-Meunier D, Chevillotte-Brivet P, di Rago JP et al: Cytochrome b-deficient mutants of the ubiquinol-cytochrome c oxidoreductase in Saccharomyces cerevisiae. Consequence for the functional and structural characteristics of the complex. J Biol Chem 1993; 268: 15626-15632.

42 Bonjardim CA, Pereira LS, Nobrega FG: Analysis of exon and intron mutants in the cytochrome $\mathrm{b}$ mitochondrial gene of Saccharomyces cerevisiae. Curr Genet 1996; 30: 200-205.

43 Torroni A, Huoponen K, Francalacci P et al: Classification of European mtDNAs from an analysis of three European populations. Genetics 1996; 144: $1835-1850$. 